\title{
ACÚMULO DE NUTRIENTES EM FRUTOS DE CAFEEIRO EM DUAS ALTITUDES DE CULTIVO: MICRONUTRIENTES ${ }^{(1)}$
}

\author{
Bruno Galvêas Laviola ${ }^{(2)}$, Herminia Emilia Prieto Martinez $^{(3)}$, Luiz \\ Carlos Chamhum Salomão(3), Cosme Damião Cruz ${ }^{(4)}$, Sebastião \\ Marcos Mendonça $^{(5)}$ \& Luciana Domiciano Silva Rosado ${ }^{(6)}$
}

\begin{abstract}
RESUMO
Dado à importância de se conhecer a exportação de micronutrientes pelos frutos, bem como, as épocas em que são mais demandados pelo cafeeiro, estudouse o acúmulo de $\mathrm{B}, \mathrm{Cu}, \mathrm{Fe}, \mathrm{Mn}$ e $\mathrm{Zn}$ em frutos de Coffea arabica $\mathrm{L}$ da antese à maturação, em lavouras estabelecidas em duas altitudes. Estudou-se também a variação no teor desses elementos. Estudou-se o acúmulo de B, Cu, Fe, Mn e Zn em frutos de cafeeiro arábico da antese à maturação em duas altitudes, bem como a variação na concentração dos elementos em folhas dos ramos produtivos. $O$ experimento foi constituído da variedade de cafeeiro (Coffea arabica L.) Catuaí IAC 44 cultivada a 720 e 950 m de altitude, no município de Martins Soares-MG. O delineamento experimental foi inteiramente ao acaso, com três repetições, usando um esquema de parcela subdividida no tempo. $O$ aumento da altitude influenciou o ciclo reprodutivo do cafeeiro, demandando maior tempo para formação dos frutos. $O$ consumo de nutrientes pelos frutos, assim como o enchimento de grãos, foi mais crítico em condições de menor altitude, já que a planta necessitou completar esses processos em menor espaço de tempo. No estádio de expansão rápida, a percentagem de acúmulo de micronutrientes foi maior na altitude de $720 \mathrm{~m}$, comparada à de $950 \mathrm{~m}$. De modo geral, a altitude influenciou a variação das concentrações foliares de nutrientes, apesar de não se ter observado resposta-padrão da concentração foliar ao aumento da altitude. Conclui-se que a altitude teve influência na extensão do ciclo, bem como no acúmulo de micronutrientes em frutos e na variação, das concentrações foliares destes elementos em folhas de cafeeiro.
\end{abstract}

Termos de indexação: fisiologia vegetal, Coffea arabica L., nutrição mineral, temperatura.

\footnotetext{
(1) Parte da Tese de Doutorado em Fitotecnia do primeiro autor. Recebido para publicação em novembro de 2006 e aprovado em julho de 2007.

(2) Engenheiro-Agrônomo, DSc., Pesquisador da Embrapa Agroenergia. CEP 70770-901 Brasília (DF). E-mail: bruno.laviola@embrapa.br

(3) Professor(a) do Departamento de Fitotecnia, Universidade Federal de Viçosa - UFV. CEP 36570-000 Viçosa (MG). E-mail: herminia@ufv.br / lsalomao@ufv.br

(4) Professor do Departamento de Biologia Geral, UFV. E-mail: cdcruz@ufv.br

${ }^{(5)}$ Engenheiro-Agrônomo, MS, Pesquisador do Centro de Pesquisas Cafeeiras Eloy Carlos Heringer - CEPEC. CEP 36900-000 Manhuaçu (MG). E-mail: sebastiao.mendonca@heringer.com.br

${ }^{(6)}$ Mestranda da Universidade Federal de Lavras - UFLA. Caixa Postal 37, CEP 37200.000 Lavras (MG). E-mail: lusrosado@yahoo.com.br
} 


\title{
SUMMARY: NUTRIENT ACCUMULATION IN COFFEE FRUITS AT TWO AT TWO PLANTATION ALTITUDES: MICRONUTRIENTS
}

\begin{abstract}
In view of the importance of knowing fruit micronutrients export from the soil, and the season in which its coffee-plant demand is higher, the accumulation of $\mathrm{B}, \mathrm{Cu}, \mathrm{Fe}, \mathrm{Mn}$ and $\mathrm{Zn}$ in fruits as well as the variation in the leaf content of the elements in productive branches of Arabic coffee was studied. The trial was performed in the period between anthesis and maturation at two altitudes. The experiment consisted of the coffee (Coffea arabica $L$.) variety Catuaí IAC 44 cultivated at 720 and $950 \mathrm{~m}$ asl, in Martins Soares, MG, Brazil. The experimental design was completely randomized with 3 repetitions using split-plots in time. The altitude affected the reproduction cycle of the coffee trees, particularly the time required for fruit formation. Micronutrient accumulation for fruits as well as grain filling are more critical at lower altitude since plants need to complete these processes in a shorter time. In the fast fruit expansion stage the percentages of micronutrient accumulation was higher in plants at $720 \mathrm{~m}$ than at $950 \mathrm{~m}$ asl. In general, the altitude influenced the variation in leaf nutrient content, although no response pattern to higher altitude was observed in the leaf concentration. It is concluded that the altitude of coffee plantations affects cycle extension, micronutrient accumulation in fruits and the variation of them in coffee plant leaves.
\end{abstract}

Index terms: plant physiology; Coffea arabica L.; mineral nutrition; temperature.

\section{INTRODUÇÃO}

Os principais micronutrientes requeridos pelo cafeeiro são B, $\mathrm{Cu}, \mathrm{Fe}, \mathrm{Mn}$ e $\mathrm{Zn}$, os quais, apesar de requeridos em pequenas quantidades, são de grande importância para o crescimento, desenvolvimento e produção do cafeeiro (Miguel et al., 2002). De modo geral, os solos adequados à cafeicultura apresentam baixa disponibilidade de alguns micronutrientes (Martinez et al., 2003a), devido à falta real ou mesmo a fatores que limitam a absorção, sendo comum a observação dos sintomas de deficiência nas lavouras (Martinez et al., 2003b).

A melhor época para fornecimento de nutrientes para suprir as exigências do crescimento e desenvolvimento dos frutos é dependente do período de maior consumo de nutrientes pelo cafeeiro (Matiello et al., 2005). Por serem os frutos os drenos preferenciais durante o período reprodutivo ( Rena \& Maestri, 1985; Carvalho et al., 1993), é importante que o suprimento de nutrientes pelas adubações anteceda os picos de acúmulo dos elementos nos frutos.

O ciclo fenológico do cafeeiro pode variar de acordo com a região em que está estabelecido, sendo mais longo em regiões de elevada altitude. Em função disso, é possível que o pico de exigência nutricional em cafeeiros plantados nessas regiões seja mais tardio que naquelas de baixa altitude. Dessa forma, as épocas e os intervalos entre as práticas de adubação deveriam ser diferenciados, levando-se em conta o período de maior exigência nutricional do cafeeiro em cada região.

Este estudo foi realizado com os objetivos de determinar a variação na concentração de $\mathrm{B}, \mathrm{Cu}, \mathrm{Fe}$, Mn e Zn nas folhas dos ramos produtivos de cafeeiro e estabelecer sua curva de acúmulo em frutos da antese à maturação, em lavouras estabelecidas em duas altitudes.

\section{MATERIAL E MÉTODOS}

O experimento foi realizado durante o período de setembro de 2005 a julho de 2006, no Centro de Pesquisas Cafeeiras Eloy Carlos Heringer (CEPEC), localizado no município de Martins Soares, MG. O experimento foi constituído da variedade de cafeeiro (Coffea arabica L.) Catuaí IAC 44 em duas altitudes (Quadro 1).

No mês de agosto de 2005 foi realizada a análise química do solo de cada talhão da propriedade (Quadro 2), a fim de efetuar a correção de acidez do solo e o fornecimento de nutrientes minerais. O fornecimento de macronutrientes foi feito via solo, dependendo da fertilidade do solo e da carga pendente de frutos (Guimarães et al., 1999) (Quadro 3). O S foi fornecido como elemento acompanhante de fertilizantes nitrogenados. Os micronutrientes $\mathrm{Zn}, \mathrm{B} \mathrm{e} \mathrm{Cu}$ foram supridos por meio de três aplicações foliares anuais aos 8, 72 e 103 dias após a antese (19/10/2005, 22/12/ 2005 e 22/01/2006, respectivamente), utilizando-se sulfato de zinco, ácido bórico, hidróxido de cobre e cloreto de potássio (como adjuvante), na concentração de $4 \mathrm{~g} \mathrm{~L}^{-1}$ de cada fertilizante.

$\mathrm{O}$ delineamento experimental empregado foi inteiramente ao acaso, distribuído em um esquema de parcelas subdividas no tempo, com duas parcelas (altitudes), 12 subparcelas (datas de amostragens) e 
Quadro 1. Caracterização das lavouras de cafeeiro Catuaí IAC 44 utilizadas no experimento

\begin{tabular}{cccc}
\hline Altitude & Idade & Espaçamento $^{\text {Produtividade }^{(2)}}$ \\
\hline & & $\mathrm{m}$ & $\mathrm{sc} \mathrm{ha}^{-1}$ \\
$720 \mathrm{~m}$ & $4 \operatorname{anos}^{(1)}$ & $2,0 \times 1,0$ & 31,60 \\
$950 \mathrm{~m}$ & 11 anos & $1,7 \times 0,7$ & 45,16 \\
\hline
\end{tabular}

(1) Lavoura recepada em 2002. ${ }^{(2)}$ Uma saca de café com $60 \mathrm{~kg}$.

Quadro 2. Características químicas e físicas dos solos da área experimental

\begin{tabular}{|c|c|c|c|c|c|c|c|c|}
\hline Altitude & MO & $\mathrm{pH} \mathrm{H}_{2} \mathrm{O}$ & $\mathbf{P}$ & $\mathbf{K}$ & $\mathrm{Ca}^{2+}$ & $\mathrm{Mg}^{2+}$ & $\mathrm{Al}^{3+}$ & $\mathrm{H}+\mathrm{Al}$ \\
\hline \multicolumn{3}{|c|}{ dag $\mathrm{kg}^{-1}$} & \multicolumn{2}{|c|}{$-\mathrm{mg} \mathrm{dm}^{-3}$} & \multicolumn{3}{|c|}{$\mathrm{cmol}_{\mathrm{c}} \mathrm{dm}^{-3}$} & + \\
\hline $720 \mathrm{~m}$ & 3,58 & 6,1 & 27,0 & 132 & 4,2 & 0,9 & 0 & 4,29 \\
\hline \multirow[t]{3}{*}{$950 \mathrm{~m}$} & 4,61 & 5,2 & 7,5 & 116 & 2,4 & 0,3 & 0 & 8,25 \\
\hline & CTC & $\mathrm{S}$ & $\mathrm{V}$ & $\mathrm{Zn}$ & $\mathrm{Fe}$ & Mn & $\mathrm{Cu}$ & B \\
\hline & $\mathrm{cmol}_{\mathrm{c}} \mathrm{dm}^{-3}$ & $\mathrm{mg} \mathrm{dm} \mathrm{m}^{-3}$ & $\%$ & 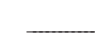 & 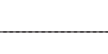 & $\mathrm{mg} \mathrm{dm}^{-3}$ & & + \\
\hline $720 \mathrm{~m}$ & 9,73 & 22,46 & 56 & 27,1 & 104,0 & 45,3 & 3,9 & 1,54 \\
\hline $950 \mathrm{~m}$ & 11,25 & 24,13 & 27 & 13,5 & 28,1 & 12,4 & 3,1 & 1,54 \\
\hline
\end{tabular}

pH em $\mathrm{H}_{2} \mathrm{O}$ : relação 1:2,5. CTC: capacidade de troca de cátions. $\mathrm{P}, \mathrm{K}, \mathrm{Fe}, \mathrm{Zn}, \mathrm{Mn}, \mathrm{Cu}$ : extrator Mehlich-1. $\mathrm{Ca}^{2+}, \mathrm{Mg}^{2+}, \mathrm{Al}^{3+}$ : extrator $\mathrm{KCl} 1 \mathrm{~mol} \mathrm{~L}^{-1}$. $\mathrm{H}+\mathrm{AL}$ : método $\mathrm{Ca}(\mathrm{OAc})_{2} 0,5 \mathrm{~mol} \mathrm{~L}^{-1}, \mathrm{pH} 7$.

três repetições. Para cada altitude foram selecionadas 20 plantas, dispostas em quatro fileiras, que constituíram as parcelas experimentais.

As amostragens iniciaram-se em 11 de outubro de 2005, quando houve antese floral, ocasião em que se coletaram folhas e flores, sendo este considerado como dia zero. A partir dessa data efetuaram-se coletas periódicas de folhas e frutos durante o desenvolvimento reprodutivo do cafeeiro: aos $34,52,65,85,100,114$, $134,161,190,217,239$ e 266 dias após a antese (DAA). Os frutos foram colhidos aleatoriamente na parcela, de ramos pertencentes ao terço médio da planta, e as folhas foram correspondentes ao terceiro e quarto pares, contadas a partir do ápice, de ramos com frutos, também situados no terço médio da planta. Foram coletados 100 frutos/parcela nas quatro primeiras amostragens, 60 frutos/parcela entre a quinta e a oitava amostragem e 20 frutos/parcela nas amostragens seguintes. As folhas foram coletadas em um número fixo de 15 folhas/parcela durante todas as amostragens.

Durante o período reprodutivo, as temperaturas mínimas e máximas, a umidade relativa (UR) entre 9 e 10 h da manhã (Quadro 4) e o índice pluviométrico (Quadro 5) foram monitorados em intervalos de tempo semanais.
Quadro 3. Adubação empregada no ano agrícola de 2005/2006

\begin{tabular}{rrrr}
\hline Altitude & $\mathbf{N}$ & $\mathbf{P}_{2} \mathbf{O}_{5}$ & $\mathbf{K}_{2} \mathbf{O}$ \\
\hline & & $\mathrm{kg} \mathrm{ha}^{-1}$ & \\
\cline { 2 - 4 } & 390 & 0 & 200 \\
$950 \mathrm{~m}$ & 370 & 0 & 70 \\
\hline
\end{tabular}

Quadro 4. Temperaturas médias das mínimas e das máximas e umidade relativa ocorridas durante o período reprodutivo do cafeeiro, em duas altitudes

\begin{tabular}{clc}
\hline \multirow{2}{*}{$\begin{array}{c}\text { Temperatura e } \\
\text { umidade }\end{array}$} & \multicolumn{2}{c}{ Altitude } \\
\cline { 2 - 3 } & $\mathbf{7 2 0} \mathbf{~ m}$ & $\mathbf{9 5 0} \mathbf{~ m}$ \\
\hline Mínima média $\left({ }^{\circ} \mathrm{C}\right)$ & 16,22 & 17,33 \\
Máxima média $\left({ }^{\circ} \mathrm{C}\right)$ & 30,22 & 26,94 \\
Umidade relativa $(\%)$ & 73,33 & 81,50 \\
\hline
\end{tabular}


Quadro 5. Índice pluviométrico mensal durante o ano agrícola 2005/06

\begin{tabular}{ccccc}
\hline \multicolumn{4}{c}{ Ano 2005 } \\
\hline Agosto Setembro Outubro Novembro & Dezembro \\
\hline & mm & & \\
\hline 12,0 & 168,4 & 59,3 & 353,5 & 278,2 \\
\hline \multicolumn{4}{c}{ Ano 2006}
\end{tabular}

Janeiro Fevereiro Março Abril Maio Junho Julho

\begin{tabular}{|c|c|c|c|c|c|c|}
\hline 7,0 & 131,0 & 256,5 & 121,8 & 31,0 & 8,0 & 13,8 \\
\hline
\end{tabular}

O material vegetal coletado foi lavado em água desionizada e posto a secar em estufa de circulação forçada de ar a $70{ }^{\circ} \mathrm{C}$ até atingir peso constante, conforme descrito por Jones Junior et al. (1991). Após esse processo, os materiais vegetais foram pesados, moídos em moinho tipo Wiley, de aço inoxidável, passados em peneira de malha de $0,841 \mathrm{~mm}^{2}$ e acondicionados em embalagens de papel devidamente identificadas, para realização das análises químicas.

Para determinação dos teores de $\mathrm{Cu}, \mathrm{Fe}, \mathrm{Mn}$ e $\mathrm{Zn}$, o material vegetal, seco e moído, foi submetido à digestão nitroperclórica (Johnson \& Ulrich, 1959), sendo quantificado por espectrofotometria de absorção atômica (AOAC, 1975). O B foi analisado após digestão das amostras por via seca (calcinação em mufla a $550{ }^{\circ} \mathrm{C}$ ) e determinado por colorimetria pelo método da Azometrina-H (Bingham, 1982).

$\mathrm{O}$ acúmulo dos micronutrientes por fruto ( $\mu \mathrm{g} /$ fruto) foi calculado pela seguinte fórmula:

Acúmulo $=\frac{\text { MS }(\mathrm{mg} / \text { fruto }) \times \text { Concentração de nutriente }\left(\mathrm{mg} \mathrm{kg}^{-1}\right)}{1.000}$

Os dados obtidos foram submetidos à análise de variância e regressão. Para explicar fisiologicamente o acúmulo de micronutrientes em frutos e a variação de nutrientes em folhas, utilizaram-se modelos de regressões sigmoidais com três e quatro parâmetros e cúbico, conforme descrito a seguir. A escolha dos modelos se deu de acordo com o ajuste $\left(\mathrm{R}^{2}\right)$ e a melhor representação do fenômeno.

$$
\begin{array}{cc}
\hat{y}=\frac{a}{1+\exp -\left(\frac{x-x_{0}}{b}\right)} \quad \hat{y}=y_{0}+\frac{a}{1+\exp -\left(\frac{x-x_{0}}{b}\right)} \\
\hat{\boldsymbol{y}}=\boldsymbol{y}_{0}+\boldsymbol{a x}+\boldsymbol{b} \boldsymbol{x}^{2}+\boldsymbol{c x ^ { 3 }} \\
\begin{array}{ll}
a=\text { ponto de máximo da curva } & \begin{array}{l}
a=\mathrm{y}_{\max }-\mathrm{y}_{\min } \\
b=\text { parâmetro de ajuste } \\
x_{0}=\text { ponto de inflexão }
\end{array} \\
x_{0}=\text { ponto de inflexão } \\
y_{0}=\text { ponto de mínimo da curva }
\end{array}
\end{array}
$$

Os pontos de inflexão das cuvas ajustadas corresponderam aos momentos em que ocorreram as taxas máximas de acúmulo de matéria seca e nutrientes em frutos $\left(\mathrm{X}_{\mathrm{TMAD}}\right)$. A taxa máxima de acúmulo diário (TMAD, $\mathrm{mg} \mathrm{dia}^{-1}$ ) foi determinada pelo acúmulo de matéria seca e nutrientes no ponto de inflexão menos o acúmulo do dia anterior.

Os pontos de curvatura mínima $\left(\mathrm{PC}_{\min }\right)$ e máxima $\left(\mathrm{PC}_{\max }\right)$ nos modelos sigmoidais foram calculados conforme método citado por Venegas et al. (1998), utilizando os parâmetros das equações não-lineares:

$$
C_{\text {min }}=x_{0}-2 b \quad C_{\max }=x_{0}+2 b
$$

$\mathrm{O} \mathrm{PC}_{\min }$ indica o momento na curva de acúmulo em que se iniciam ganhos significativos no acúmulo de micronutrientes. Já o $\mathrm{PC}_{\max }$ indica o momento em que o acúmulo dos elementos começa a se estabilizar.

$\mathrm{O}$ acúmulo relativo (ARe) foi obtido de acordo com as diferenças entre o mínimo e o máximo acúmulo em cada estádio de formação do fruto, sendo os valores em percentagem obtidos em relação ao acúmulo final alcançado.

$$
\begin{gathered}
A R e=A_{F}-A_{I} \\
\text { ARe }=\text { acúmulo relativo no estádio de } \\
\text { formação do fruto } \\
\text { A Fe A I = acúmulo no final e início do estádio } \\
\%=\frac{A R e}{A T} * 100 \\
\text { AT = acúmulo alcançado aos } 266 \text { DAA }
\end{gathered}
$$

Calculou-se a diferença entre os teores foliares no início do ciclo reprodutivo, na época da floração $\left(\mathrm{C}_{\mathrm{F}}\right)$, em relação às concentrações foliares de micronutrientes em meados dos estádios de expansão rápida $\left(\mathrm{C}_{\mathrm{ER}}\right.$, 87 DAA a 720 m e 79,5 DAA a $950 \mathrm{~m}$ ) e granação $\left(\mathrm{C}_{\mathrm{G}}\right.$, 162,5 DAA a $720 \mathrm{~m}$ e $188 \mathrm{DAA}$ a $950 \mathrm{~m}$ ). Esse gradiente de concentração indica se houve competição fruto/folha pelo acúmulo de micronutrientes nos momentos de maior exigência nutricional da cultura.

\section{RESULTADOS E DISCUSSÃO}

A altitude de cultivo da lavoura influenciou a duração dos estádios reprodutivos, bem como a extensão do ciclo do cafeeiro. Na altitude de $720 \mathrm{~m}$, observou-se que o fruto necessitou de 211 dias para sua formação, enquanto a $950 \mathrm{~m}$ a formação foi até 262 dias após a antese (DAA) (Laviola et al., 2007). É provável que a ocorrência de menores temperaturas máximas (Quadro 4) tenha influenciado as reações enzimáticas e o transporte de fotoassimilados (Larcher, 2004), ampliando o tempo de formação do fruto de cafeeiro. A duração do ciclo nas duas altitudes foi maior comparada à observada por Laviola (2004) em 
Viçosa-MG, o qual verificou ciclo de 224 dias para formação dos frutos de cafeeiro. Entretanto, Viçosa está a $650 \mathrm{~m}$ de altitude, o que evidencia a redução da extensão do ciclo do cafeeiro em menores altitudes.

Os frutos de cafeeiro passaram por cinco estádios distintos de formação: chumbinho, expansão rápida, crescimento suspenso, granação e maturação. Cada estádio de formação possui funções fisiológicas e metabólicas próprias, essenciais à formação final da semente de café (Laviola et al., 2007).

As curvas de acúmulo de micronutrientes em frutos de cafeeiro seguiram um modelo sigmóide simples, com exceção para o $\mathrm{Cu}$ a $720 \mathrm{~m}$ e o $\mathrm{Mn}$ a $950 \mathrm{~m}$ de altitude (Figura 1). Geralmente o padrão das curvas de micronutrientes em frutos difere do padrão de acúmulo de matéria seca em frutos, o qual segue um modelo de sigmóide dupla (Coombe, 1976; Rena \& Maestri, 1985).

No estádio de chumbinho os frutos apresentam baixa taxa de crescimento (Laviola et al., 2007), que, segundo Cannel (1971) e Leon \& Fournier (1962), pode ser decorrente da elevada taxa respiratória e de multiplicação celular, não se observando efeito da altitude no acúmulo de micronutrientes nos frutos de cafeeiro neste estádio (Figura 1; Quadros 6, 8 e 9). É possível que os efeitos da altitude, principalmente os relacionados à temperatura (Quadro 4), influenciem menos nas primeiras semanas de formação do fruto de cafeeiro. De modo geral, a ascensão no acúmulo de micronutrientes $\left(\mathrm{PC}_{\min }\right.$, Quadro 7) apresentou tendência de começar antes do início do estádio de expansão rápida. Isso indica que as demandas metabólicas por micronutrientes nos frutos iniciamse um pouco antes do estádio de expansão rápida, no qual se observam incrementos significativos de matéria seca nos frutos (Cannel, 1971; Coombe, 1976).

Pode-se observar, no estádio de chumbinho, que a proporção (\%) de acúmulo, tanto de B como de Zn, nos frutos foi maior, quando comparada à de acúmulo dos outros micronutrientes (Quadro 8). É provável que o maior acúmulo de B e Zn no estádio de chumbinho esteja relacionado à grande importância destes nutrientes nos processos de divisão celular e na estabilização de membranas das novas células formadas (Marschner, 1995; Marenco \& Lopes, 2005).

O estádio de expansão rápida se caracteriza, principalmente, por rápido alongamento das células dos frutos, atingindo cerca de $80 \%$ do seu tamanho final. $\mathrm{O}$ acúmulo de matéria seca no estádio de expansão rápida está relacionado, principalmente, à expansão celular, com deposição de material de parede, sendo essencial o fluxo de água para os frutos no processo de alongamento celular. Provavelmente, o acúmulo de nutrientes nos frutos no estádio de expansão ocorra por fluxo de massa decorrente das altas taxas de translocação de água para os frutos neste estádio, tão necessária para expansão celular (Laviola et al., 2007).
No estádio de expansão rápida, a altitude influenciou o acúmulo de micronutrientes nos frutos (Quadro 8), observando-se, proporcionalmente, maiores acúmulos relativos (ARe) de B, Cu, Fe, Mn e Zn na altitude de $720 \mathrm{~m}$, em relação à de $950 \mathrm{~m}$. Os maiores valores da TMAD na altitude de $720 \mathrm{~m}$ (Quadro 7) concorreram para que houvesse maior acúmulo de nutrientes no final do estádio de expansão nesta altitude. As temperaturas máximas (Quadro 4) mais elevadas ocorridas na altitude de $720 \mathrm{~m}$ possivelmente contribuíram para que houvesse maiores taxas de translocação de micronutrientes para os frutos neste estádio.

Dentre todos os micronutrientes, o $\mathrm{Zn}$ foi o elemento que apresentou maior acúmulo relativo (\%) no estádio de expansão rápida, denotando a importância deste na síntese de triptofano, aminoácido precursor da biossíntese da auxina, ácido indol acético (AIA), essencial para o processo de alongamento celular (Marschner, 1995).

O estádio de crescimento suspenso caracteriza-se por desaceleramento no crescimento dos frutos de cafeeiro, apresentando baixas taxas de acúmulo de matéria seca (Rena et al., 2001). No entanto, em geral, o acúmulo de micronutrientes manteve-se contínuo neste estádio de crescimento (Figura 1; Quadros 6 e 9). Neste estádio, acredita-se que esteja havendo reciclagem e síntese de enzimas e compostos intermediários (Taiz \& Zeiger, 2004), antes empregados na síntese de polímeros de parede, para serem utilizados como precursores na síntese de compostos de reservas no estádio de granação. Pelo fato de os micronutrientes terem papéis importantes na ativação de enzimas (Marschner, 1995), é possível que o acúmulo contínuo deles no estádio de crescimento suspenso tenha ocorrido em função do aumento da demanda metabólica desses elementos.

No estádio de granação, também chamado de enchimento do endosperma, a matéria seca é depositada, sobretudo, nas sementes (Laviola et al., 2007). A estabilização $\left(\mathrm{PC}_{\max }\right)$ no acúmulo de micronutrientes no estádio de granação ocorreu em menor tempo na altitude de $720 \mathrm{~m}$ (Quadro 8), ou seja, a altitude teve influência na velocidade de transporte dos micronutrientes para os frutos. Além disso, o acúmulo de nutrientes estabilizou-se antes do final do estádio de granação, ou seja, primeiro são acumulados os nutrientes para depois completarem-se os processos finais de formação das sementes. O consumo de nutrientes pelos frutos, assim como o enchimento de grãos, é mais crítico em condições de menor altitude, já que a planta necessita completar esses processos em menor espaço de tempo.

$\mathrm{OX}_{\mathrm{TMAD}}$ nas duas altitudes ocorreu entre meados do estádio de expansão rápida do fruto e início do estádio de granação deste, dependendo do micronutriente avaliado (Quadro 7). Apesar de a altitude não ter influenciado diretamente o $\mathrm{X}_{\mathrm{TMAD}}$, $\mathrm{o}$ mesmo não ocorreu em relação ao TMAD, sendo observados valores maiores na altitude de $720 \mathrm{~m}$. 

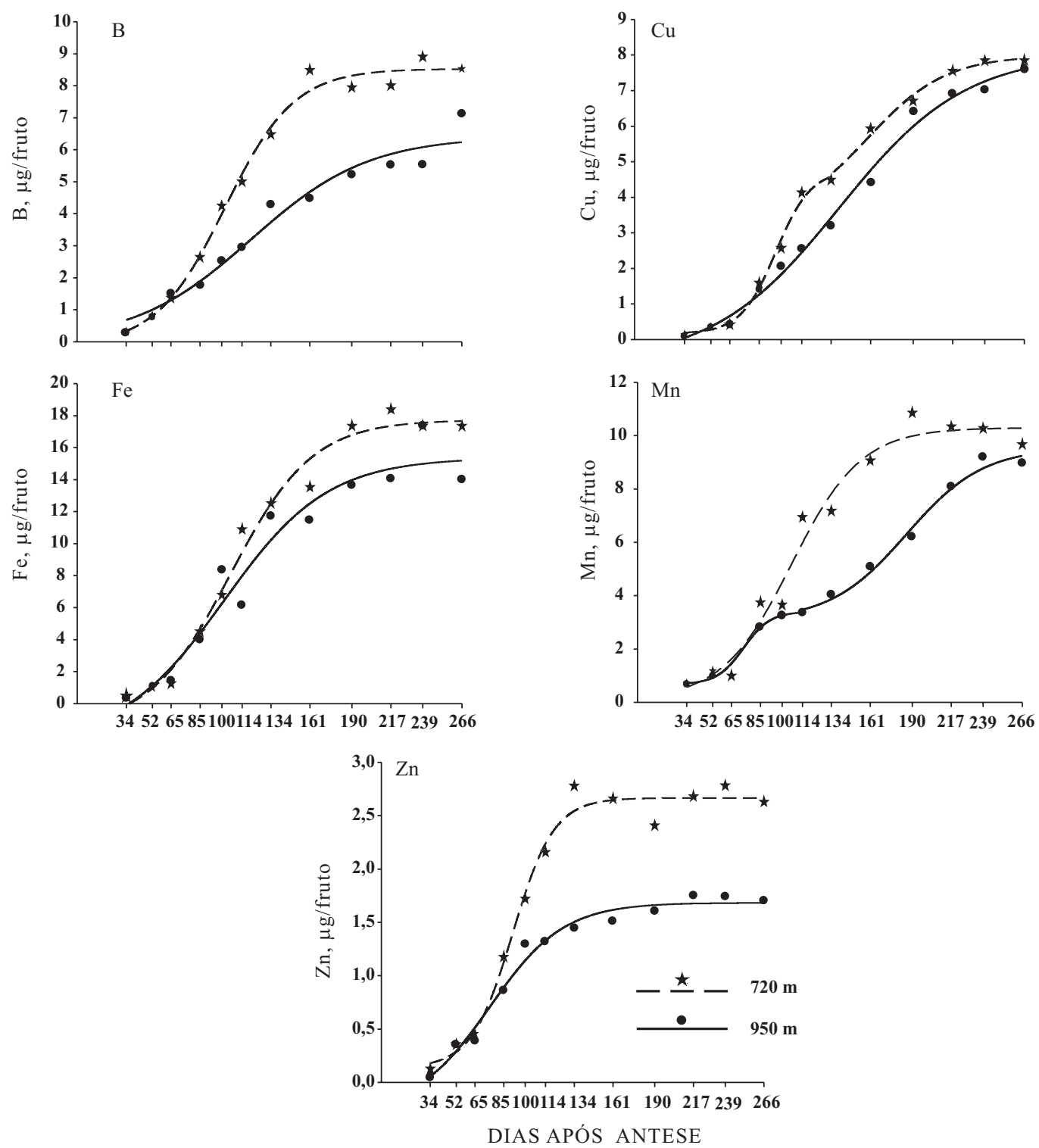

Figura 1. Acúmulo de boro, cobre, ferro, manganês e zinco em frutos de cafeeiro em função do tempo decorrido após a antese, em duas altitudes.

O acúmulo relativo no estádio de granação (Quadro 8) foi maior na altitude de $950 \mathrm{~m}$, comparada à de $720 \mathrm{~m}$. Isso ocorreu, principalmente, em razão de o acúmulo de micronutrientes na altitude de $720 \mathrm{~m}$ ter se antecipado nas primeiras fases de formação do fruto.

Após o estádio de granação iniciou-se o de maturação, que se caracteriza, principalmente, pelo aumento do teor de açúcares e por mudanças na coloração da casca do fruto (Puschmann, 1975; Rena et al., 2001). Apesar de o estádio de maturação (Quadro 6) ter perdurado até 266 DAA, época em que foi realizada a última amostragem, na altitude de $720 \mathrm{~m}$ a mudança na coloração da casca do fruto de verde para vermelho ocorreu em menor tempo - fato observado visualmente.
Os efeitos da altitude no acúmulo de $\mathrm{B}, \mathrm{Cu}, \mathrm{Fe}$, Mn e Zn estão relacionados, sobretudo, a variações de temperaturas máximas entre as altitudes (Quadro 4). De acordo com Larcher (2004), a temperatura possui influência direta sobre processos regulatórios da planta. Sob menores temperaturas, a velocidade das reações enzimáticas é reduzida e, conseqüentemente, as taxas fotossintéticas e respiratórias também são restringidas - isso também desfavorece a absorção de nutrientes pelas raízes.

Em temperaturas mais elevadas, aumenta-se a velocidade de difusão e de fluxo em massa de nutrientes para as raízes; a penetração de nutrientes no espaço livre aparente via apoplasto estimula sua absorção e seu acúmulo nas células das raízes e favorece o transporte nos condutos do xilema. Além disso, o 
Quadro 6. Equações de regressão do acúmulo de micronutrientes em frutos de cafeeiros em função do tempo decorrido após a antese

\begin{tabular}{|c|c|c|c|c|}
\hline Nutriente & Altitude & Período (DAA) & Modelo & $\mathbf{R}^{2}$ \\
\hline & & dia & & \\
\hline \multirow[t]{2}{*}{ B } & $720 \mathrm{~m}$ & $34-266$ & $\hat{y}=-0,1336+8,6642 /(1+\exp (-(\mathrm{x}-102,3467) / 23,9163))$ & 0,990 \\
\hline & $950 \mathrm{~m}$ & $34-266$ & $\hat{y}=6,4097 /(1+\exp (-(\mathrm{x}-120,4642) / 40,5818))$ & 0,964 \\
\hline \multirow[t]{3}{*}{$\mathrm{Cu}$} & $720 \mathrm{~m}$ & $34-134$ & $\hat{y}=0,1694+4,6018 /(1+\exp (-(\mathrm{x}-96,4392) / 12,0166))$ & 0,992 \\
\hline & & $114-266$ & $\hat{y}=3,3409+4,6470 /(1+\exp (-(\mathrm{x}-159,1127) / 26,7152))$ & 0,992 \\
\hline & $950 \mathrm{~m}$ & $34-266$ & $\hat{y}=-0,6381+8,6831(1+\exp (-(\mathrm{x}-139,6711) / 43,0022))$ & 0,993 \\
\hline \multirow[t]{2}{*}{$\mathrm{Fe}$} & $720 \mathrm{~m}$ & $34-266$ & $\hat{y}=-1,4979+19,2159 /(1+\exp (-(\mathrm{x}-106,1629) / 28,2750))$ & 0,985 \\
\hline & $950 \mathrm{~m}$ & $34-266$ & $\hat{y}=-2,4301+17,7910 /(1+\exp (-(\mathrm{x}-101,7280) / 35,7521))$ & 0,9481 \\
\hline \multirow[t]{3}{*}{$\mathrm{Mn}$} & $720 \mathrm{~m}$ & $34-266$ & $\hat{y}=0,0996+10,1966 /(1+\exp (-(x-106,6915) / 240336))$ & 0,976 \\
\hline & $950 \mathrm{~m}$ & $34-114$ & $\hat{y}=0,6776+2,7235 /(1+\exp (-(\mathrm{x}-73,3391) / 9,1945))$ & 0,999 \\
\hline & & $100-266$ & $\hat{y}=3,0761+6,4690 /(1+\exp (-(\mathrm{x}-186,0927) / 26,4541))$ & 0,989 \\
\hline \multirow[t]{2}{*}{$\mathrm{Zn}$} & $720 \mathrm{~m}$ & $34-266$ & $\hat{y}=0,1354+2,5302 /(1+\exp (-(\mathrm{x}-91,3274) / 14,3091))$ & 0,986 \\
\hline & $950 \mathrm{~m}$ & $34-266$ & $\hat{y}=-0,2669+1,9509 /(1+\exp (-(\mathrm{x}-75,4248) / 25,6446))$ & 0,982 \\
\hline
\end{tabular}

Quadro 7. Ponto da taxa máxima de acúmulo diário ( $\left.\mathrm{X}_{\mathrm{TMAD}}, \mathrm{DAA}\right)$, taxa máxima de acúmulo diária (TMAD) e pontos de curvatura mínima $\left(\mathrm{PC}_{\min }, \mathrm{DAA}\right)$ e máxima $\left(\mathrm{PC}_{\mathrm{max}}, \mathrm{DAA}\right)$, em duas altitudes

\begin{tabular}{|c|c|c|c|c|c|c|}
\hline Nutriente & Altitude & Período & $\mathrm{X}_{\mathrm{TMAD}}$ & TMAD & $\mathbf{C}_{\min }$ & $\mathrm{C}_{\max }$ \\
\hline & & 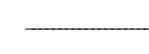 & - & $\mu \mathrm{g} /$ fruto & 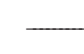 & - \\
\hline \multirow[t]{2}{*}{ B } & $720 \mathrm{~m}$ & $34-266$ & 102 & 0,091 & 55 & 150 \\
\hline & $950 \mathrm{~m}$ & $34-266$ & 120 & 0,039 & 39 & 202 \\
\hline \multirow[t]{3}{*}{$\mathrm{Cu}$} & $720 \mathrm{~m}$ & $34-134$ & 96 & 0,096 & 72 & 120 \\
\hline & & $114-266$ & 159 & 0,043 & 106 & 213 \\
\hline & $950 \mathrm{~m}$ & $34-266$ & 139 & 0,051 & 54 & 225 \\
\hline \multirow[t]{2}{*}{$\mathrm{Fe}$} & $720 \mathrm{~m}$ & $34-266$ & 106 & 0,170 & 50 & 163 \\
\hline & $950 \mathrm{~m}$ & $34-266$ & 101 & 0,124 & 30 & 173 \\
\hline \multirow[t]{3}{*}{$\mathrm{Mn}$} & $720 \mathrm{~m}$ & $34-266$ & 106 & 0,106 & 59 & 155 \\
\hline & $950 \mathrm{~m}$ & $34-114$ & 73 & 0,074 & 55 & 92 \\
\hline & & $100-266$ & 186 & 0,061 & 133 & 239 \\
\hline \multirow[t]{2}{*}{$\mathrm{Zn}$} & $720 \mathrm{~m}$ & $34-266$ & 91 & 0,044 & 63 & 120 \\
\hline & $950 \mathrm{~m}$ & $34-266$ & 75 & 0,019 & 34 & 127 \\
\hline
\end{tabular}


Quadro 8. Acúmulo relativo (ARe) de acordo com o estádio de formação do fruto de cafeeiro, em duas altitudes

\begin{tabular}{|c|c|c|c|c|c|c|c|c|c|c|c|}
\hline \multirow{2}{*}{ Nutriente } & \multirow{2}{*}{ Altitude } & \multicolumn{2}{|c|}{ Chumbinho } & \multicolumn{2}{|c|}{ Exp. rápida } & \multicolumn{2}{|c|}{ Cresc. susp. } & \multicolumn{2}{|c|}{ Granação } & \multicolumn{2}{|c|}{ Maturação ${ }^{(1)}$} \\
\hline & & ARe & $\%$ & ARe & $\%$ & ARe & $\%$ & ARe & $\%$ & ARe & $\%$ \\
\hline \multirow[t]{2}{*}{$\mathrm{B}$} & $720 \mathrm{~m}$ & 1,369 & 16,07 & 3,428 & 40,23 & 0,436 & 5,12 & 3,206 & 37,62 & 0,082 & 0,96 \\
\hline & $950 \mathrm{~m}$ & 1,203 & 19,29 & 1,137 & 18,23 & 0,610 & 9,79 & 3,269 & 52,42 & 0,017 & 0,28 \\
\hline \multirow[t]{2}{*}{$\mathrm{Cu}$} & $720 \mathrm{~m}$ & 0,483 & 6,11 & 3,091 & 39,11 & 0,331 & 4,18 & 3,500 & 44,28 & 0,499 & 6,32 \\
\hline & $950 \mathrm{~m}$ & 0,563 & 7,40 & 1,118 & 15,61 & 0,694 & 9,13 & 5,123 & 67,34 & 0,040 & 0,53 \\
\hline \multirow[t]{2}{*}{$\mathrm{Fe}$} & $720 \mathrm{~m}$ & 2,146 & 12,15 & 6,456 & 36,55 & 0,841 & 4,76 & 7,825 & 44,31 & 0,393 & 2,23 \\
\hline & $950 \mathrm{~m}$ & 1,884 & 12,41 & 4,118 & 27,12 & 1,975 & 13,01 & 7,185 & 47,32 & 0,021 & 0,14 \\
\hline \multirow[t]{2}{*}{$\mathrm{Mn}$} & $720 \mathrm{~m}$ & 1,629 & 15,84 & 3,814 & 37,09 & 0,525 & 5,10 & 4,198 & 40,82 & 0,118 & 1,15 \\
\hline & $950 \mathrm{~m}$ & 1,242 & 13,43 & 1,985 & 21,47 & 0,142 & 1,54 & 5,829 & 63,06 & 0,046 & 0,50 \\
\hline \multirow[t]{2}{*}{$\mathrm{Zn}$} & $720 \mathrm{~m}$ & 0,482 & 18,09 & 1,913 & 60,53 & 0,140 & 5,23 & 0,430 & 16,13 & 0,001 & 0,02 \\
\hline & $950 \mathrm{~m}$ & 0,441 & 26,22 & 0,671 & 39,87 & 0,217 & 12,91 & 0,353 & 21,00 & 0,001 & 0,01 \\
\hline
\end{tabular}

${ }^{(1)}$ Considerou-se como maturação o período após o início da estabilização no acúmulo de MS no fruto, embora as mudanças na coloração da casca correspondentes à maturação tenham se iniciado antes.

aumento da temperatura acelera a atividade respiratória da planta e incrementa o metabolismo nas raízes e a produção de ATP, liberando energia, que é utilizada de diversos modos para absorção de íons (Marenco \& Lopes, 2005).

Além de influenciar os processos de absorção, a temperatura também pode alterar a taxa de transporte de nutrientes, assim como a partição de fotoassimilados no floema. Segundo Taiz \& Zeiger (2004), o resfriamento de um tecido-dreno inibe as atividades que necessitam de energia metabólica e resulta na diminuição da velocidade do transporte em direção ao dreno. De acordo com Rena \& Maestri (1985), a temperatura ótima para assimilação de $\mathrm{CO}_{2}$ no cafeeiro varia de 20 a $30^{\circ} \mathrm{C}$, dependendo da temperatura em que as plantas foram aclimatadas nos dias anteriores.

O fornecimento de micronutrientes ao cafeeiro, seja via solo ou via folha (Guimarães et al., 1999; Rena \& Favaro, 2000), deve se iniciar antes do estádio de expansão rápida do fruto. Nas condições experimentais, o fornecimento de $\mathrm{B}, \mathrm{Cu}$ e $\mathrm{Zn}$ deveria ocorrer antes dos 34 DAA (9/11/2005). Se a aplicação de micronutrientes for via solo, ela deve ser efetuada após a florada, pelo fato de as fontes de micronutrientes apresentarem, em geral, baixa taxa de liberação.

Se o fornecimento dos micronutrientes for via folha, ele poderá ser efetuado em torno dos 30 DAA, já que a absorção via folha e a distribuição dos micronutrientes nas plantas são processos mais rápidos (Rena \& Favaro, 2000).
Calculando as médias dos momentos em que se estabiliza o acúmulo de B, Cu e Zn em frutos, verificase que estes momentos ocorrem aos 160 DAA a $720 \mathrm{~m}$ e 185 DAA na altitude de $950 \mathrm{~m}$. Considerando três pulverizações anuais para fornecimento dos micronutrientes (Matiello et al., 2005) e que a última pulverização deva ocorrer pelo menos 20 dias antes do fim do acúmulo de micronutrientes, pode-se fazer a seguinte sugestão de aplicação foliar: efetuar três pulverizações anuais, sendo a primeira realizada aos 30 DAA, independentemente da localização da lavoura; a segunda e a terceira devem ocorrer com intervalos de 30-35 dias em lavouras estabelecidas em altitudes próximas a $720 \mathrm{~m}$ e intervalos de 40-45 dias em cafezais estabelecidos em altitudes em torno de $950 \mathrm{~m}$. Independentemente da altitude, a concentração de Zn na calda deve ser em proporção superior nas duas primeiras pulverizações.

Dentre os três principais micronutrientes, o Zn é o único que não pode ser fornecido eficientemente via solo, devido à sua grande facilidade em ser adsorvido pelos colóides (Rena \& Favaro, 2000). Em caso de fornecimento do $\mathrm{Zn}$ via foliar isoladamente, sugere-se que as pulverizações ocorram com intervalos de 25 a 30 dias, independentemente da altitude.

A concentração de micronutrientes B, Fe e Zn no terceiro e quarto pares de folhas de ramos produtivos ao longo do ciclo reprodutivo do cafeeiro (Figura $2 \mathrm{e}$ Quadro 9) mostrou-se menor no início do período reprodutivo, durante o estádio de expansão rápida do fruto, quando foi atingida concentração mínima. 

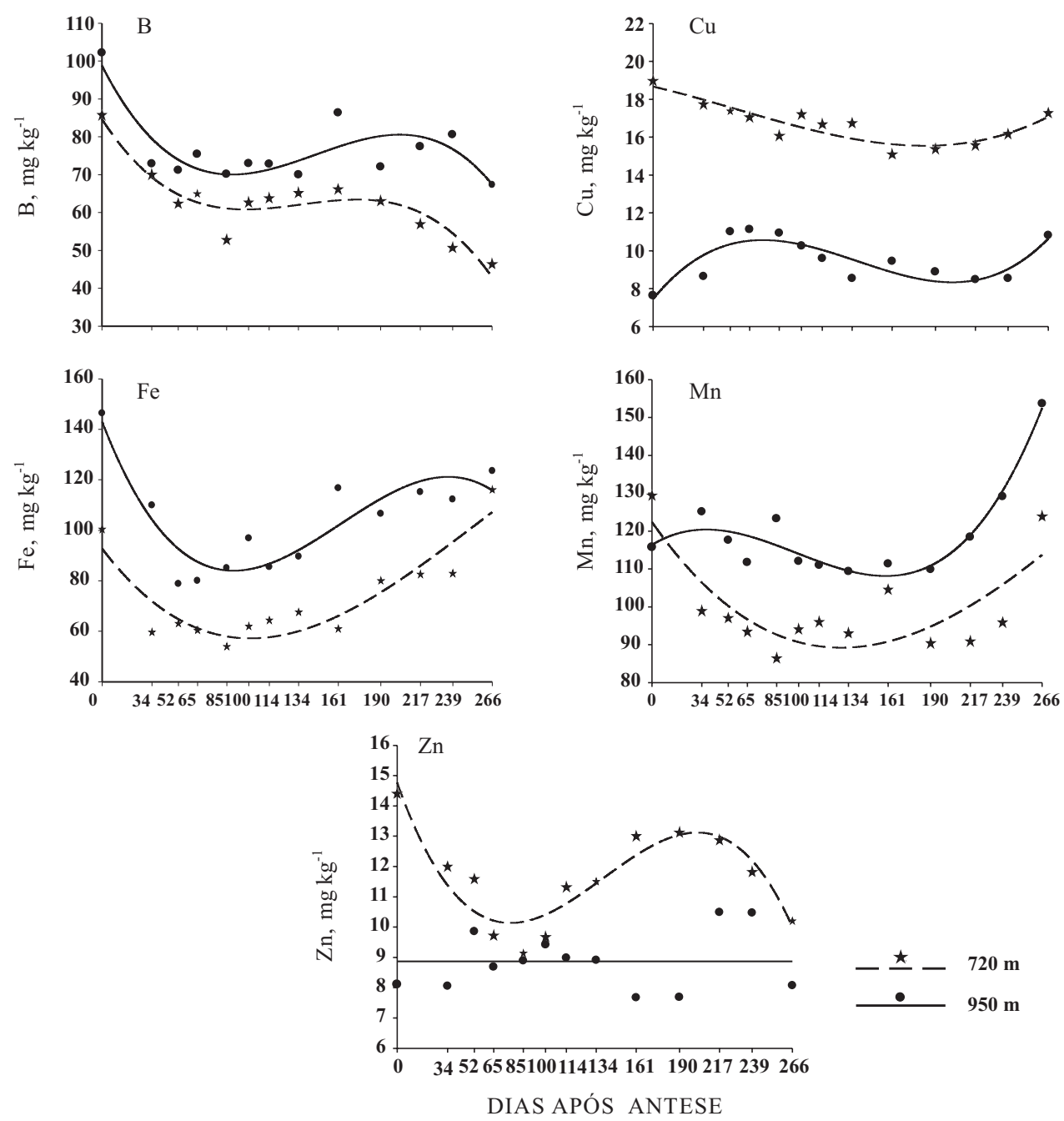

Figura 2. Variação na concentração de boro, cobre, ferro, manganês e zinco em folhas de cafeeiro em função do tempo decorrido após a antese, em duas altitudes.

Quadro 9. Equações de regressão da variação nas concentrações de micronutrientes em folhas de cafeeiros em função do tempo decorrido após a antese, em duas altitudes - ponto de mínimo $\left(X_{\min }\right)$ e máximo $\left(X_{\text {max }}\right)$

\begin{tabular}{|c|c|c|c|c|c|}
\hline Nutriente & Altitude & Modelo & $\mathbf{R}^{2}$ & $\mathbf{X}_{\min }$ & $\mathbf{X}_{\max }$ \\
\hline \multirow{3}{*}{$\mathrm{B}$} & & & & $-\mathrm{c}$ & - \\
\hline & $720 \mathrm{~m}$ & $\hat{y}=84,6806-0,6003 x+0,0048 x^{2}-0,0000117 x^{3}$ & 0,872 & 266 & 0 \\
\hline & $950 \mathrm{~m}$ & $\hat{y}=98,8114-0,7604 \mathrm{x}+0,0062 \mathrm{x}^{2}-0,0000141 \mathrm{x}^{3}$ & 0,739 & 266 & 0 \\
\hline \multirow[t]{2}{*}{$\mathrm{Cu}$} & $720 \mathrm{~m}$ & $\hat{y}=18,6776-0,0183 x-0,0000808 x^{2}+0,000000477 x^{3}$ & 0,815 & 183 & 0 \\
\hline & $950 \mathrm{~m}$ & $\hat{y}=7,4351+0,0964 x-0,0009 x^{2}+0,00000216 x^{3}$ & 0,738 & 0 & 266 \\
\hline \multirow[t]{2}{*}{$\mathrm{Mn}$} & $720 \mathrm{~m}$ & $\hat{y}=122,4093-0,5578 \mathrm{x}+0,0027 \mathrm{x}^{2}-0,00000258 \mathrm{x}^{3}$ & 0,631 & 101 & 266 \\
\hline & $950 \mathrm{~m}$ & $\hat{y}=116,3268+0,2381 x-0,0040 x^{2}+0,0000134 x^{3}$ & 0,917 & 90 & 0 \\
\hline \multirow[t]{2}{*}{$\mathrm{Fe}$} & $720 \mathrm{~m}$ & $\hat{y}=92,7241-0,7361 \mathrm{x}+0,0047 \mathrm{x}^{2}-0,00000615 \mathrm{x}^{3}$ & 0,839 & 129 & 122 \\
\hline & $950 \mathrm{~m}$ & $\hat{y}=143,0347-1,5084 x+0,0116 x^{2}-0,0000238 x^{3}$ & 0,818 & 159 & 266 \\
\hline \multirow[t]{2}{*}{$\mathrm{Zn}$} & $720 \mathrm{~m}$ & $\hat{y}=14,7820-0,1393 x+0,0013 x^{2}-0,00000301 x^{3}$ & 0,849 & 266 & 0 \\
\hline & $950 \mathrm{~m}$ & $\hat{y}=\bar{y}=8,8641$ & - & & \\
\hline
\end{tabular}


Quadro 10. Concentrações foliares de micronutrientes na floração $\left(C_{F}\right)$, comparada às concentrações foliares em meados dos estádios de expansão rápida $\left(C_{E R}\right)$ e granação $\left(C_{G}\right)$, em duas altitudes

\begin{tabular}{|c|c|c|c|c|c|c|}
\hline \multirow[t]{2}{*}{ Nutriente } & \multirow[t]{2}{*}{ Altitude } & \multirow[t]{2}{*}{ Florada } & \multicolumn{2}{|c|}{ Crescimento rápido } & \multicolumn{2}{|c|}{ Granação } \\
\hline & & & & $\begin{array}{r}\mathrm{C}_{\mathrm{ER}}-\mathrm{C}_{\mathrm{F}} \\
-\mathrm{mg} \mathrm{kg}^{-1}\end{array}$ & & $\mathrm{C}_{G}-\mathrm{C}_{F}$ \\
\hline \multirow[t]{2}{*}{ B } & $720 \mathrm{~m}$ & 84,68 & 60,98 & $-23,69$ & 63,25 & $-21,43$ \\
\hline & $950 \mathrm{~m}$ & 98,81 & 70,23 & $-28,57$ & 80,06 & $-18,74$ \\
\hline \multirow[t]{2}{*}{$\mathrm{Cu}$} & $720 \mathrm{~m}$ & 18,67 & 18,78 & $-1,88$ & 15,61 & $-3,05$ \\
\hline & $950 \mathrm{~m}$ & 7,43 & 10,54 & 3,12 & 8,39 & 0,97 \\
\hline \multirow[t]{2}{*}{$\mathrm{Fe}$} & $720 \mathrm{~m}$ & 92,72 & 57,76 & $-34,96$ & 66,51 & $-26,20$ \\
\hline & $950 \mathrm{~m}$ & 143,03 & 84,55 & $-58,47$ & 111,83 & $-31,20$ \\
\hline \multirow[t]{2}{*}{$\mathrm{Mn}$} & $720 \mathrm{~m}$ & 122,41 & 92,325 & $-30,08$ & 90,961 & $-31,45$ \\
\hline & $950 \mathrm{~m}$ & 116,33 & 116,99 & 0,66 & 110,49 & $-5,84$ \\
\hline \multirow[t]{2}{*}{$\mathrm{Zn}$} & $720 \mathrm{~m}$ & 14,78 & 10,19 & $-4,58$ & 12,42 & $-2,35$ \\
\hline & $950 \mathrm{~m}$ & 8,86 & 8,86 & - & 8,86 & - \\
\hline
\end{tabular}

Quanto ao $\mathrm{Cu}$ e Mn, eles apresentaram mínima concentração nas folhas no estádio de granação. Durante todo o período reprodutivo, observou-se que as concentrações de B, Fe e Mn nas folhas permaneceram maiores na altitude de $950 \mathrm{~m}$, comparada à de $720 \mathrm{~m}$. Isso pode ter ocorrido em razão da competição pela translocação dos elementos, já que os frutos na altitude de $720 \mathrm{~m}$ apresentaram maior acúmulo final dos nutrientes (Figura 1).

No quadro 10 encontra-se a comparação das concentrações foliares em meados dos estádios de expansão rápida e de granação com as concentrações foliares observa no início do ciclo reprodutivo (floração). Essas comparações indicam se houve competição, ao longo do ciclo reprodutivo, pela translocação dos micronutrientes. De modo geral, observou-se que a presença dos frutos resultou em forte competição fruto/ folha pela partição dos micronutrientes, independentemente do estádio de formação dos frutos. Apesar disso, as concentrações de micronutrientes ao longo do período reprodutivo estiveram na maior parte do tempo dentro das faixas de referência estipuladas por Martinez et al. (2004).

\section{CONCLUSÕES}

1. A altitude influenciou o acúmulo de micronutrientes em frutos e folhas de cafeeiro.

2. A altitude de $720 \mathrm{~m}$ contribuiu para maior velocidade no acúmulo de micronutrientes em frutos.
3. A concentração de micronutrientes nas folhas de cafeeiro não foi influenciada apenas pela altitude de cultivo, mas também por outros fatores que podem influenciar a taxa de distribuição de nutrientes na planta.

\section{AGRADECIMENTOS}

À Universidade Federal de Viçosa, ao CNPq e ao Consórcio Brasileiro de Pesquisa e Desenvolvimento do Café, pelo apoio financeiro.

\section{LITERATURA CITADA}

ASSOCIATION OF OFFICIAL ANALYTICAL CHEMISTS AOAC. Official methods of analysis. 12.ed. Washington, 1975. 1094p.

BINGHAM, F.T. Boron. In: PAGE, A.L., ed. Methods of soil analysis: Chemical and microbiological properties. Madison, American Society of Agronomy, 1982. p.431447. (Serie Agronomy, 9)

CANNEL, M.G.R. Seasonal patterns of growth and development of arabica coffee in Kenya. Part 4. Effects of seasonal differences in rainfall on bean size. Kenya Coffee, 36:175-180, 1971.

CARVALHO, C.H.S.; RENA, A.B.; PEREIRA, A.A. \& CORDEIRO, A.T. Relação entre produção, teores de N, $\mathrm{P}, \mathrm{Ca}, \mathrm{Mg}$, amido e seca de ramos do Catimor (Coffea arabica L.). Pesq. Agropec. Bras., 28:665-673, 1993. 
COOMBE, B.G. The development of fleshy fruits. Ann. Rev. Plant Physiol., 27: 507-528, 1976.

GUIMARÃES, P.T.G.; GARCIA, A.W.R.; ALVAREZ V., V.H.; PREZOTTI, L.C.; VIANA, A.S.; MIGUEL, A.E.; MALAVOLTA, E.; CORREAA, J.B., LOPES, A.S.; NOGUEIRA, F.D. \& MONTEIRO, A.V.C. Cafeeiro. In: RIBEIRO, A.C.; GUIMARÃES, P.T.G. \& ALVAREZ V., V.H., eds. Recomendações para o uso de corretivos e fertilizantes em Minas Gerais, $5^{\mathrm{a}}$ Aproximação. Viçosa, MG, Comissão de Fertilidade do Solo do Estado de Minas Gerais - CFSEMG, 1999. p.289-302.

JOHNSON, C. M. \& ULRICH, A. Analytical methods for use in plants analyses. Los Angeles, University of California, 1959. v.766. p.32-33.

JONES JUNIOR, J.B.; WOLF, B. \& MILLS, H.A. Plant analysis handbook: A practical sampling, preparation, analysis and interpretation guide. Athens, Micro Macro Publishing, 1991. 213p.

LARCHER, W. Ecofisiologia vegetal. São Carlos, RiMa, 2004. $531 \mathrm{p}$.

LAVIOLA, B.G. Dinâmica de macronutrientes em folhas, flores e frutos de cafeeiro arábico em três níveis de adubação. Viçosa, MG, Universidade Federal de Viçosa, 2004. 100p. (Tese de Mestrado)

LAVIOLA, B.G.; MARTINEZ, H.E.P.; SALOMÃO, L.C.C.; CRUZ, C.D. \& MENDONÇA, S.M. Acúmulo de nutrientes em frutos de cafeeiro em quatro altitudes de cultivo: Cálcio, magnésio e enxofre. R. Bras. Ci. Solo, 31:1451-1462, 2007.

LEON, J. \& FOURNIER, L. Crecimento y desarollo del fruto de Coffea arabica. Turrialba, 12:65-74, 1962.

MARENCO, R.A. \& LOPES, N.F. Fisiologia vegetal: Fotossíntese, respiração, relações hídricas e nutrição mineral. Viçosa, MG, Universidade Federal de Viçosa, 2005. 451p.

MARSCHNER, H. Mineral nutrition of higher plants. 2.ed. New York, Academic Press, 1995. 889p.

MARTINEZ, H.E.P ; NEVES, Y.P. \& ZABINI, A.V. Diagnóstico do estado nutricional do cafeeiro. In: ZAMBOLIM, L., ed. Produção integrada de café. Viçosa, MG, Universidade Federal de Viçosa, 2003b. p.397-1442.
MARTINEZ, H.E.P.; MENEZES, J.F.S.; SOUZA, R.B.; ALVAREZ V., V.H. \& GUIMARÃES, P.T.G. Faixas críticas de concentrações de nutrientes e avaliação do estado nutricional de cafeeiros em quatro regiões em Minas Gerais. Pesq. Agropec. Bras., 38:703-713, 2003a.

MARTINEZ, H.E.P.; SOUZA, R.B.; ALVAREZ V., V.H.; MENEZES, J.F.S.; NEVES, Y.P.; OLIVEIRA, J.A.; ALVARENGA, A.P. \& GUIMARÃES, P.T.G. Nutrição mineral, fertilidade do solo e produtividade de cafeeiro nas regiões de Patrocínio, Manhuaçu, Viçosa, São Sebastião do Paraíso e Guaxupé. 2.ed. Belo Horizonte, EPAMIG, 2004. 60p. (EPAMIG Boletim Técnico, 72)

MATIELLO, J.B.; SANTINATO, R.; GARCIA, A.W.R.; ALMEIDA, S.R. \& FERNANDES, D.R. Cultura de café no Brasil: Novo manual de recomendações. Rio de Janeiro, MAPA /PROCAFE, 2005. 438p.

MIGUEL, A.E.; MATIELLO, J.B.; VIANA, A.S. \& JAPIASSU, L. Teores de micronutrientes nas amostras de solo analisadas pelo laboratório do mapa em Varginha, Sul de Minas. In: CONGRESSO BRASILEIRO DE PESQUISAS CAFEEIRAS, 28., Caxambu, 2002. Anais. Rio de Janeiro, MAPA/PROCAFÉ, 2002. p.76-77.

PUSCHMANN, R. Características bioquímicas do fruto do cafeeiro (Coffea arabica L.) durante a maturação. Viçosa, MG, Universidade Federal de Viçosa, 1975. 35p. (Tese de Mestrado)

RENA, A.B. \& FÁVARO, J.R.A. Nutrição do cafeeiro via folha. In: ZAMBOLIM, L., ed. Café: Produtividade, qualidade e sustentabilidade. Viçosa, MG, Universidade Federal de Viçosa, 2000. p.149-208.

RENA, A.B. \& MAESTRI, M. Fisiologia do cafeeiro. Inf. Agropec., 11:26-40, 1985.

RENA, A.B.; BARROS, R.S. \& MAESTRI, M. Desenvolvimento reprodutivo do cafeeiro. In: ZAMBOLIM L., ed. Tecnologias de produção de café com qualidade. Viçosa, MG, Universidade Federal de Viçosa, 2001. p.101-128.

TAIZ, L. \& ZEIGER, E. Fisiologia vegetal. 3.ed. Porto Alegre, Artmed, 2004.719p.

VENEGAS, J.G.; HARRIS, R.S. \& SIMON, B.A. A comprehensive equation for the pulmonary pressurevolume curve. J. Appl. Physiol., 84:389-395, 1998. 